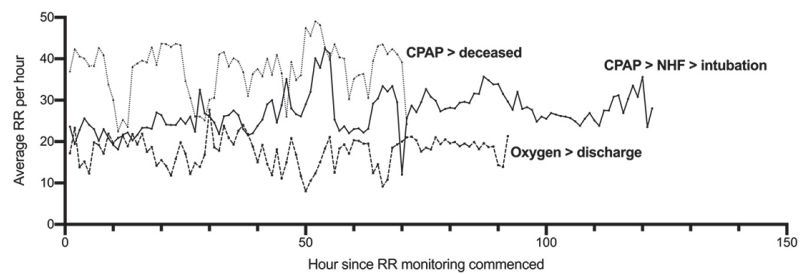

Abstract P24 Figure 1 CARP trial wearable respiratory rate, respiratory support and outcome data from 3 patients with severe COVID-19

required. Wearable RR sensors have been benchmarked against reference sensors. They offer the potential for continuous remote monitoring at scale of patients at risk for deterioration, who may require escalation and respiratory support. In the CARP trial we are exploring feasibility and utility of continuous wearable RR monitoring of inpatients with COVID19 , with the potential of extrapolating this to other causes of respiratory failure in the future.

Method The pre-commercial chest-worn Altair PneumoWave derives continuous RR and detects apnoeas, with processing algorithms benchmarked with reference impedance plethsmography. The device attaches to a standard adhesive ECG 'dot' with bluetooth hub and wi-fi connectivity. Live RR and event data is presented in a co-designed cloud-based dashboard, allowing for remote visualisation and inpatient journey tracking.

Consenting inpatients with PCR-confirmed COVID-19 who required oxygen therapy were screened over an 8-month period. Follow-up was performed at 28 and 90-days post discharge, including post-COVID patient reported outcomes and MRC dyspnoea score.

Results 156 patients were screened, with 77 recruited to the CARP trial. 32 patients required non-invasive respiratory support, of which 14 were escalated to mechanical intubation. 17 patients died within trial.

Bland-Altman analyses of paired RR data confirmed that wearable sensor data shows good agreement with critical care RR monitoring (Phillips Intellivue MX700), and that ward-based intermittent clinician $\mathrm{RR}$ measurements were imprecise.

From the initial utility review of CARP physiology data visualisations, rising hourly average $\mathrm{RR}>25 / \mathrm{min}$ is associated with subsequent patient deterioration. Improving and stable hourly average RR of $<25 / \mathrm{min}$ associates with stable respiratory failure and improvement to hospital discharge (figure 1).

Conclusion Continuous wearable respiratory rate remote monitoring in COVID-19 inpatients is feasible. Planned machine learning and time-series analyses of the detailed physiology and clinical endpoint data will determine appropriate cut-offs and feature importance for deteriorating patient risk predictions. The CARP clinical dashboard provides an infrastructure for future implementation and evaluation of these AI insights.

\section{P25 THE EFFECT OF POST COVID-19 REHABILITATION ON HEALTH STATUS USING THE EQ-5D- $5 \mathrm{~L}$}

${ }^{1} \mathrm{~T}$ Williamson, ${ }^{1} \mathrm{~F}$ Dyer, ${ }^{2} \mathrm{D}$ Garvey, ${ }^{1} \mathrm{~A}$ Miers, ${ }^{3} \mathrm{C}$ Morris, ${ }^{1} \mathrm{C}$ Wells, ${ }^{1} \mathrm{~S}$ Rahman. ${ }^{1}$ Epsom and St Helier University Hospitals NHS Trust, Epsom, UK; ${ }^{2}$ Sutton Health and Care, Sutton, UK; ${ }^{3}$ Surrey Downs Health and Care, Leatherhead, UK

10.1136/thorax-2021-BTSabstracts. 135
Introduction Many patients who have survived COVID-19 are left with ongoing health concerns; be it deconditioning from a prolonged hospital admission or those with long COVID syndrome. Rehabilitation programmes have been developed in attempt to address this but little is understood about its effectiveness. The EQ-5D-5L is a well validated measure of health status and was completed before and after rehabilitation. We aimed to investigate the effect of our rehabilitation programme on health status.

Method All patients attending assessment for post-COVID rehabilitation complete the EQ-5D-5L and is repeated on discharge. Patients attended rehabilitation twice a week for 6 weeks.

Results Between July 2020 and May 2021136 patients completed post-COVID rehabilitation. Mean age was 56 (12.25). $38 \%$ male. Table 1 illustrates change pre and post rehabilitation.

Abstract P25 Table 1 Mean (SD) scores for EQ-5D-5L index pre and post rehabilitation

\begin{tabular}{llll}
\hline Pre rehabilitation & Post rehabilitation & change & P value* $^{*}$ \\
\hline $0.611(0.195)$ & $0.733(0.172)$ & $0.127(0.187)$ & $<0.05$ \\
\hline Paired t-test & & &
\end{tabular}

Conclusion Post COVID rehabilitation improves health status in patients following COVID-19 with ongoing health concerns.

\section{P26 KNOWLEDGE SEEKING BEHAVIOUR OF THE COVID-19 POPULATION. ANALYSIS OF THE FIRST MILLION UK USERS OF YOUR COVID RECOVERY ${ }^{\circledR}$}

${ }^{1}$ RE Barclay, ${ }^{1} \mathrm{~N}$ Gardiner, 'E Chaplin, 'A Watt, ' $\mathrm{G}$ Mills, ${ }^{1} \mathrm{M}$ Baldwin, ${ }^{1} \mathrm{~K}$ Hicklin, ${ }^{1,2} \mathrm{~S}$ J Singh, on behalf of the multi-disciplinary national Your COVID Recovery core team. ' University Hospitals Leicester, Leicester, UK; ${ }^{2}$ University of Leicester, Leicester, UK

\subsection{6/thorax-2021-BTSabstracts. 136}

Introduction University Hospitals Leicester NHS Trust (UHL) in partnership with NHS England worked in collaboration with professional societies, charities and healthcare professionals (HCPs) nationwide to launch the Your COVID Recovery website (www.yourcovidrecovery.nhs.uk). This site offers information on living with and recovering from the physical and psychological effects of COVID-19. The site has information on the commonly reported post COVID symptoms ${ }^{1}$ and was launched in July 2020. The aim was to evaluate website usage and the knowledge seeking behaviour of its readers.

Method The first one million users were analysed using Google Analytics from July 2020 to February 2021, reporting on overall site usage, content page views and length of time spent on the pages. The top three content pages were recorded each month.

Results To reach one million users took seven months, with over 700,000 of these users viewing the site from December 2020 to February 2021.

Retrieval of content data (figure 1) shows that Fatigue was the most viewed page $(225,511)$ followed by Musculoskeletal Shoulder and Back Pain (MSK) (212,312) and Cough $(184,178)$. Fatigue consistently featured every month, whereas Musculoskeletal Shoulder and Back Pain and Cough did not feature until October 2020 and November 2020 respectively. 